\title{
Memetics and Pragmatic Cognition
}

\author{
Li Ran \\ College of Basic Science \\ Tianjin Agricultural University \\ Tianjin, China \\ nature618class@126.com
}

\begin{abstract}
This paper first introduces the definition of meme, and then analyzes its features and factors which play a significant part in its replication, spread, selection and elimination. After that the author studies and speculates the cognition process of language meaning and proposes the hypothesis of "pragmatic processing models" in the frame of memetics. Last the author highlights the necessity of experimental pragmatic study of the cognitive processing models.
\end{abstract}

Keywords-meme; language meaning; cognitive processing; pragmatic cognition

\section{The Definition of Meme}

Memetics is the theoretical and empirical science that studies the replication, spreading and evolution of memes [1]. The term and concept of "meme" were firstly introduced by an Oxford professor Richard Dawkins in his book The Self Gene in 1976. The root of the term "meme" comes from "mimneskesthai", the Greek term for "memory" and the structure of "meme" is coined by analog with "gene". There are different definitions of "meme". Grant defines it as "a contagious information pattern that replicates by parasitically infecting human minds and alternating their behavior, causing them to propagate the pattern [2].” Tony Lezard regards it as simply "a unit of intellectual or cultural information that survives long enough to be recognized as such, and which can pass from mind to mind [3]." Grant clarifies that "an idea can parasitically infect your mind and alter your behavior, causing you to want to tell your friends about the ideas, thus exposing them to the idea-virus [2]. Any idea which does this is called a meme." Heylighen explains, "Meme is an information pattern, held in an individual's memory, which is capable of being copied to another individual's memory [1]." Blackmore stresses that "memes are anything that is copied from person to person, and in the modern world the vast majority of memes are words, and combinations of words, both written and spoken [4]." Dawkins assumes the way meme works is that "just as genes propagate themselves in the gene pool by leaping from body to body via sperms or eggs, so memes propagate themselves in the meme pool by leaping from brain to brain via a process which, in the broad sense, can be called imitation [5].” Different definitions generally and roughly agree that a meme is some sort of self-replicating and selfpropagating unit of cultural transmission with the main method of imitation. Simply speaking, the term "meme" often refers to any piece of information passed from one mind to

1. The Ministry of Education of Humanities and Social Science Project 2018.

2. China Language Assessment (No.ZGWYCPJJ2018028B )

3. Major Education Reform Funds of Tianjin Agricultural University (2017-E-00). another. The common examples are ideas, songs, proverbs, slogans, fashion elements, ways of thinking, speaking and doing, cultures, traditions, customs and language, etc.

\section{The Replication of Meme}

Memes are supposed to have evolution through natural selection in a similar way to Darwin's theory on biological evolution. That is "they are subject to replication, mutation, survival and competition [6]". So it is important to emphasize that memes are supposed to spread as being the most effective replicators. Not all the information can be copied, only the selected ones can be memes. People choose what to copy and whom to imitate. Let us suppose that there are a dozen different greeting styles around that compete with each other. Now it is important for any individual to choose the right one to copy. The right decision is the one that benefits the greeting style memes themselves. These memes spread whenever they get the chance. Perhaps the simplest one "hi" wins out and becomes an effective replicator and propagates rapidly to express greeting in the most efficient way. It needs not actually be simple, or formal, or imitable, or fashionable, whatever, but as long as it is the fittest one to survive and spread.

Another factor influencing the replicator selection is whom to imitate, which is more influential than what is copied. That is "copy the most successful" strategy. Success, status and popularity are a matter of being able to be chosen and copied. Blackmore calls these people "meme fountains". "They provide a plentiful source of memes--both old memes they have copied and new memes they have invented by building on, or combining, the old [4]". That is why western suit becomes globalized, Christmas is celebrated outside Europe and America and "cool", "sorry" and "ok", etc. can be heard in nearly every local language.

In a word, the memetic success depends on the feature and survivability of memes, such as easy to remember, or easy to pronounce, but also on whom is copied. According to P.F. Jenkins, meme is a selective imitation and replication device. Moreover, this imitation and replication often occurs with variation or mutation [5]. He observes a kind of island bird near New Zealand good at singing some nine different songs. The same song sung by birds of different generation sounds different, because the song is not inherited but imitated by the young birds with slice deviation. Such is the same case with other memes, like language. 


\section{The Application of Memetics to Language Cognition}

Language as a form of information could be considered as memeplex when it is transmitted and replicated as a comprehensive package. Applying the memetics to language, Liu Si proposes, "One meaningful utterance could be a 'meaning-memeplex"' [7]. She refers the utterance meaning as a core that mostly accompanied by a context in reality; it is with the linguistic forms serving to express and interpret the meaning. The meaning-memeplex would be relatively stable, but also dynamic. During the replication, meaning-memeplex would do adjustment, such as alteration, addition to and/or removal from the elements in the candidate meaningmemeplex according to the situational and/or linguistic information needed, to interpret the speaker's meaning correctly. She also proposes that the processing of utterance meaning can be classified into two distinguished phases: "zero experienced" ("to experience" refers "to imitate, to learn, to acquire) and "experienced"; one transforming stage: "experiencing". In the "zero experienced" phase, it is assumed that one would not be able to interpret the utterance that he has neither experienced nor simulated. In the "experiencing" stage, one would simulate an old alike meaning-memeplex for a new meaning-memeplex. He would finally simply replicate or copy the meaning-memeplex in the "experienced" phase.

Based on Liu's proposal, when people encounter a word or an utterance for the first time, they would simulate a comparable meaning-memeplex by "experiencing” [7]. For example, I was confused when I heard someone said, "You have a hollow leg." But after experiencing (learning), which is a simulation of a certain existing meaning-memeplex (like "eat a lot"), I saw that it was a way to say, "You really can eat a lot." Similarly, someone talked about his college life and said that some of his friends even organized a band, technically a band. I then comprehended that his friends' band was equipped with advanced technology. Later I knew "technically" here meant "so called". The band was so suck that it could not be regarded as a qualified band at all. Then I stored these sentences with the context together in my mind. People store tens of thousands of words in memory not isolated but with context. Generally speaking, context can be divided into cultural context, situational context and linguistic context [6]. Yet, I see the context in two ways-cultural context and linguistic context which are partially consistent with "semantic context" and "pragmatic context".

As to culture context, when Chinese people encounter “地主”, they intuitively treat it negatively and hard to take it equal to “landowner”. When “条 $\square$ ” comes into one’s sight, especially those familiar with Chinese history, it is often enriched as "unequal treaty" , and "favorable treaty" can seldom come into Chinese people’s mind. Also “明星” is always or even exclusively regarded as "film stars" or

"TV stars" . "Company stars" or "political stars" can never be given top priority. In fact, these words are cultureloaded. They entail rich culture connotations. It is almost impossible to comprehend it as it is without any cultural implication. These words and culture are co-existing and inseparable. They are processed as a whole.
Secondly, linguistic context which refers to the words and sentences around also plays an important role in word and sentence cognition. The following test might be able to show the importance of linguistic context. "The story making you laugh is call joke, the meat of pig is called pork, the tool you use for meal is called fork, and so the white part of an egg is called__." "Yolk" will be the intuitive answer of most people even though they can suddenly realize the mistake after a second thought. Linguistic context has an effect on your mind. An article in U.S News \& World Report (2003/09/19) says “Aoccdrnig to rseearch at a Cmabdirge uinervtisy, it deosn't mttaer in waht oredr the ltteers in a wrod are. The olny iprmoetnt tihng is taht frist and lsat ltteers are at the rghit pclae. The rset can be a toatl mses, and you can sitll raed it wouthit a porbelm. Tihs is bcuseae we do not raed ervey lteter by itslef but the wrod as a wlohe." (According to research at a Cambridge University, it doesn't matter in what order the letters in a word are. The only important thing is that first and last letters are at the right place. The rest can be a total mess, and you can still read it without a problem. This is because we do not read every letter by itself but the word as a whole.) Also we read the sentence or even the paragraph as a whole. Each word can be recognized easier and faster in sentence and in paragraph than in isolation. Linguistic context does have a positive effect on word and sentence cognition. My statement is in line with Liu Si's sample experiments where she found that when students were asked to write out their interpretations of isolated sentences, they produced utterance meanings with "various imagined contexts."

\section{The Hypothesis of LANGuage Cognition Process}

I assume that language cognition process might be divided into three stages, which could not be explained by a uniform theory. Processing models are different from stage to stage. I predict that at stage 1 where people come across certain utterance for the first time, linguistic meaning should be processed first. If the linguistic interpretation makes no sense and then the hearer test the linguistic meaning against its context to get the contextual and implied meaning. For example, the linguistic meaning of "Can you tell me the time?" is "Do you have the ability to tell me the time of the present moment". Obviously in most daily situations it is not the information conveyed by the speaker. So it is an indirect request for time information. Take “吃了 $\square$ ? ” as a Chinese example. Its literal meaning will be first processed, say by a foreigner, and found insufficient to figure out the speaker's intention when the hearer meets the speaker perhaps on a street and be greeted like this for the first time. Later he/she will find out that it is a Chinese way of greeting (after others' explanation or by his/her own contemplation). Then he/she stores this greeting meme with this context (memeplex) in mind. So stage 1 would be consistent with the Literal-First Serial Model proposed by Gricean which assumes that a hearer must first recover the minimal proposition expressed by an utterance before engaging in any further pragmatic processing [8]. When the hear encounters the meme for the second, the third or even the fourth time, he/she is at stage 2, and that is to say the literal meaning and the context of an utterance come out of one's mind simultaneously, but the contextual meaning is preferred to the literal one and chosen 
as the utterance meaning. So if the same hearer is greeted “吃 了 $\square$ ? ” next time, he/she can recognize that it is a greeting expression even though he/she finds it strange. As time goes by, he/she frequently be greeted so and becomes quite familiar with this greeting. He/she also adopts it to greet others. The hearer becomes the host of the memeplex. He/she is at stage 3. Due to the high frequency of replication and imitation, the pragmatic interpretations of such sentence like “吃了 $\square$ ? ” or "Can you tell me the time?" are standardized (as greeting or request form). This is partially consistent with the "standardized" proposed by Bach and Harnish [9]. At stage 3, contextual meaning is always processed first. But whether literal meaning is processed or not remains questionable. The more standardized an expression becomes, the less literal meaning is processed. But there is no absolute standardized sentence, so there is no clear-cut between stage 2 and 3 . The processing of "what is said" and "what is implicated", happens at stage 2 and 3 .

In fact, language cognitive process is more complicated than the ideal model. I then assume that people may encounter the same utterance many times in many situations. Each situation may endow the utterance a unique contextual meaning. This utterance with various contexts it situated in and the relative contextual meanings are stored in people's mind as a whole. The following chart gives us a clear view.

Utterance $\begin{gathered}\text { context } 1 \text {--- utterance meaning } 1 \\ \text { context } 2 \text {--- utterance meaning } 2 \\ \text { context } 3 \text {--- utterance meaning } 3\end{gathered}$

If the utterance occurs in context 1 , then utterance meaning 1 is triggered. If the utterance occurs in context 1 with a very high frequency, then meaning 1 is always quoted. Utterance meaning 1 is in the tendency of standardization. But that does not mean that people never process meaning 2 and 3 . If context permitted, meaning 2 and 3 will be triggered and come out. For example,

Utterance: “吃了吗?” (Have you had meal?)

Context 1: After dinner, you take a walk on the street. Your neighbor sees you and says, "Have you had dinner?"

Meaning 1: Greeting, meaning “hi”.

Context 2: You work late and return home at 20:00. The moment you enter your house, your wife says, "Have you had dinner?”

Meaning 2: Question, meaning "Have you already had dinner tonight or not since you work so late?”

Context 3: You pay a visit to your friend and find that his family are having terrific dinner. Your friend says, "Have you had dinner?"

Meaning 3: Invitation, meaning "Would you like to have dinner with us?”

The above three contexts do not occur with equal frequency. Context 1 is commonly encountered. So meaning 1 is always triggered to the extent that it becomes standardized. But when people happen to be in context 2 and 3 , they can recover meaning 2 (the literal meaning) and meaning 3 (another implied meaning). But whether their effort for meaning 1 's recovery is the same as that for meaning 2 remains questionable. One thing I am sure is that the latest perceived contextual meaning can affect the recovery efforts. When the target context is similar to the newly encountered context, the recovery efforts can be reduced. When different, the efforts are enhanced. If people meet brand-new context that does not belong to the stored ones, then a new chain will be added as "utterance--context 4-meaning 4". If an existed context seldom encountered or never be encountered from then on, the corresponding meaning will be forgotten and eliminated. According to Heylighen's proposal, "the memeplex life-cycle consists of 4 stages that collaboratedly work to determine the memeplex fitness (the overall survival and proliferation rate of a meme) [1]". The four subsequent stages are: "(1) assimilation by an individual, who thereby becomes a host of the memeplex; (2) retention in that individual's memory; (3) expression by the individual that can be perceived by others; (4) transmission of the memeplex thus created a new memeplex vehicle to one or more other individuals. This last stage is followed again by stage 1 , thus closing the replication loop. At each stage there is selection, meaning that some memes will be eliminated”.

Of course, there are few contexts that are exactly the same. So the utterance meaning is triggered by the most similar context stored in the hearer's cognitive system. There is also a possibility that the given context is similar to two kinds of stored contexts and people could not judge which one is the right one immediately. Then two contextual meaning will be triggered together. For example, you work late and return home at 20:00. You meet your next-door neighbor on your way home. He says to you, "Have you had dinner?" this context is similar to both context 1 and 2 mentioned above. So at that moment, contextual meaning 1 (greeting) and meaning 2 (a real question) are triggered perhaps at the same time. They compete with each other to fit the given context. The winner is the fittest memeplex. By further contextual hint such as the speaker's rising tone and serious and careful facial expression, meaning 2 (a real question) would be selected. In most cases, several meanings might be processed simultaneously. For example, "Is Julia at home?” can be used in its literal sense to ask a question, a direct speech act. On the telephone it can also be used as a request to call Julia to the phone, an indirect speech act. When it is constructed literally, it elicits such responses as "Yes, she is" or "No, she isn't". But when it is constructed as an indirect request, it leads to a simple request, like "I'll get her" or "Just a minute", which responds to the indirect meaning alone. In other contexts it leads to two-part responses, like "Yes, she is---I'll get her", the first part of which, "Yes, she is", answers the literal question, and the second part, "I'll get her", responds to the indirect request. The selection of a contextual meaning or meanings mainly depends on the similarity between the given memeplex and the memeplexs stored in people's cognitive system, on the frequency of replication of the memeplex and on the newly perceiving of the memeplex. 


\section{CONCLUSION AND Discussion}

Memetics provides us a new way to study and speculate the cognitive process of human language. The replication, spread, selection and elimination of language which is a form of meme are supposed to share the same feature with gene. Therefore, with the help of meme study (memetics), the hypothesis of language cognition process models are about to come into being.

But still this hypothesis of "meaning-memeplex" to language cognition needs experimental investigation and support. Later we will focus on the teating of proposed cognition models.

\section{ACKNOWLEDGEMENT}

This work was financially supported by The Ministry of Education of Humanities and Social Science Project 2018 "The Study of Chinese Figurative Language Processing-Eyetracking Experiments on Chinese Irony and Metaphor”, China Language Assessment (No.ZGWYCPJJ2018028B) and Major Education Reform Funds of Tianjin Agricultural University "Research and Practice on College English Module Teaching" (2017-E-00).

\section{REFERENCES}

[1] Heylighen, F. Memetics [OL]. http://pespmc1.vub.ac.be/MEMES.html, 2001a.

[2] Grant, G. Memetic Lexicon [OL]. http://pespmc1.vub.ac.be/MEMLEX.html, 1990.

[3] Lezard, T. What is A Meme [OL]. http://www.ejhs.org/volume9/coon.htm, 2006.

[4] Blackmore, S. Four Commentaries in Perspectives on Imitation: From Mirror Neurons to Memes [OL]. http://www.susanblackmore.co.uk/Chapters/Imit2005.htm, 2005.

[5] Dawkins, R. The Selfish Gene [M]. Oxford: Oxford University Press, 1976.

[6] Gao Feng. A discussion on the method of discourse analysis in source language [J]. Education Exploration, 2006(5):42-44. (In Chinese)

[7] Liu, S. "Meaning-memeplex" in Cognitive Mechanism of Chinese Language in Use [R]. A proposal to the 10th International Pragmatics Conference-GÖteborg, Sweden, 2006.

[8] Bezuidenhout, A and Morris R.K. Implicature, Relevance and Default Pragmatic Inference [A]. Ira, A and Dan Sperber. Experimental pragmatics [C]. Britain: Antony Rowe Ltd, Chippenham and Eastbourne, 2004, 228-253.

[9] Bach, K., \& Harnish, R. Linguistic Communication and Speech Acts [M]. Cambridge, MA: MIT Press, 1979. 